\title{
\begin{tabular}{l|l} 
Mitraries & DSpace@MIT
\end{tabular}
}

\author{
MIT Open Access Articles
}

\section{Unsteady Adjoint of Pressure Loss for a Fundamental Transonic Turbine Vane}

The MIT Faculty has made this article openly available. Please share how this access benefits you. Your story matters.

Citation: Talnikar, Chaitanya, Qiqi Wang, and Gregory M. Laskowski. “Unsteady Adjoint of Pressure Loss for a Fundamental Transonic Turbine Vane." Volume 5A: Heat Transfer (June 13, 2016).

As Published: http://dx.doi.org/10.1115/GT2016-56689

Publisher: ASME International

Persistent URL: http://hdl.handle.net/1721.1/116206

Version: Final published version: final published article, as it appeared in a journal, conference proceedings, or other formally published context

Terms of Use: Article is made available in accordance with the publisher's policy and may be subject to US copyright law. Please refer to the publisher's site for terms of use. 


\section{UNSTEADY ADJOINT OF PRESSURE LOSS FOR A FUNDAMENTAL TRANSONIC TURBINE VANE}

\author{
Chaitanya Talnikar; Qiqi Wang \\ Aerospace Computational Design Lab \\ Department of Aerospace and Astrophysics \\ Massachusetts Institute of Technology \\ Cambridge, Massachusetts 02139 \\ talnikar@mit.edu, qiqi@mit.edu
}

\author{
Gregory M. Laskowski \\ Engineering Technologies \\ GE Aviation \\ Lynn, Massachusetts, 01910 \\ laskowski@ge.com
}

\begin{abstract}
High fidelity simulations, e.g., large eddy simulation are often needed for accurately predicting pressure losses due to wake mixing and boundary layer development in turbomachinery applications. An unsteady adjoint of high fidelity simulations is useful for design optimization in such aerodynamic applications. In this paper we present unsteady adjoint solutions using a large eddy simulation model for a vane from VKI using aerothermal objectives. The unsteady adjoint method is effective in capturing the gradient for a short time interval aerothermal objective, whereas the method provides diverging gradients for long timeaveraged thermal objectives. As the boundary layer on the suction side near the trailing edge of the vane is turbulent, it poses a challenge for the adjoint solver. The chaotic dynamics cause the adjoint solution to diverge exponentially from the trailing edge region when solved backwards in time. This results in the corruption of the sensitivities obtained from the adjoint solutions. An energy analysis of the unsteady compressible Navier-Stokes adjoint equations indicates that adding artificial viscosity to the adjoint equations can dissipate the adjoint energy while potentially maintain the accuracy of the adjoint sensitivities. Analyzing the growth term of the adjoint energy provides a metric for identifying the regions in the flow where the adjoint term is diverging. Results for the vane from simulations performed on the Titan supercomputer are demonstrated.
\end{abstract}

\footnotetext{
${ }^{*}$ Address all correspondence to this author.
}

\section{NOMENCLATURE}

$\bar{p}_{l} \quad$ Pressure loss objective

$\bar{p}_{t, l} \quad$ Mass-averaged stagnation pressure loss on downstream plane

$\bar{p}_{t, \text { in }} \quad$ Stagnation pressure at the inlet

$p_{t, p} \quad$ Stagnation pressure on downstream plane

$T$ Time-averaging interval

$S$ Surface area of boundary

$V \quad$ Volume of domain

$\rho_{p}$ Density on downstream plane

$p_{p} \quad$ Pressure on downstream plane

$\gamma$ Isentropic expansion factor

$M_{p} \quad$ Mach number on downstream plane

$\mathbf{x}$ Position vector

$D$ Domain for the fluid problem

$\rho$ Density at a point in the domain

u Velocity vector

$c$ Speed of sound

$E$ Total energy

$\mu$ Viscosity coefficient

$\alpha$ Thermal coefficient

$e$ Internal energy

$\sigma$ Viscous stress tensor

w Conservative variables vector

F Navier-Stokes convective flux vector

$F^{v}$ Navier-Stokes viscous flux vector

$\bar{J}$ Time-averaged objective

$J$ Instantaneous objective 
$\hat{\mathbf{w}}$ Adjoint of conservative variables

A Jacobian of convective flux 3-dimensional tensor

$\mathbf{A}^{\mathbf{v}} \quad$ Jacobian of viscous flux 3-dimensional tensor

D Jacobian of viscous flux with respect to gradient terms 4dimensional tensor

s Navier-Stokes equations source term vector

q Primitive variables vector

v Symmetrized variables vector

S Transformation matrix from primitive to symmetrized variables

T Transformation matrix from conservative to symmetrized variables

V Transformation matrix from conservative to primitive variables

Â Primitive flux 3-dimensional tensor for symmetrized variables

$\hat{\mathbf{v}}$ Adjoint for symmetrized variables

B Jacobian of $\hat{\mathbf{A}}$ with respect to symmetrized variables

$\hat{\mathbf{D}}$ Viscous 4-dimensional tensor

$E_{\hat{v}} \quad L_{2}$ norm of adjoint of symmetrized variables

$M, M_{1}, M_{2}$ Growth matrix of adjoint energy

$a, b \quad$ Scaled speeds of sound

$\sigma_{1} \quad$ Maximum singular value of growth matrix

$\lambda$ Scaling factor for additional adjoint viscosity

$L \quad$ Length scale of fluid problem

\section{INTRODUCTION}

High fidelity simulations like large eddy simulations (LES) are essential for accurately simulating turbulent fluid flows. This is especially true for turbomachinery applications in which there is a transitioning boundary layer and flow separation. Gourdain [1] compared LES to low fidelity methods like Reynolds averaged Navier-Stokes simulations (RANS) and found that LES predicts heat transfer with a much higher accuracy when analyzed against experimental data. Moreover, LES is becoming more feasible with the growing power of supercomputers. In just over a decade compute capacity has increased by a factor of 100. This has enabled high fidelity simulations for fluid problems where the Reynolds number is on the order of a million.

For accomplishing design of turbomachinery components using LES in a reasonable amount of time it is necessary to obtain gradients of design objectives with respect to design parameters. A straightforward method to obtain gradients is to use finite difference, but the number of simulations required scales linearly with the number of input parameters. An alternative is to use the adjoint method, which provides the gradient with respect to a large number of parameters using just one additional simulation. This method has been extensively used for performing design optimization using steady-state Euler [2] or RANS [3] simulations. The adjoint method involves solving a set of equations known as the adjoint equations. For a time-dependent simulation like
LES an unsteady adjoint method is required in which the adjoint equations are simulated backwards in time to obtain the desired derivatives. Recently, Economon [4] performed unsteady adjoint simulations for a rotating airfoil, but these were restricted to unsteady laminar fluid flows.

It has been observed in numerous studies $[5,6]$ and through simulations conducted for this paper, that for turbulent fluid flows the unsteady adjoint solution grows exponentially when simulated backwards in time. This is due to the chaotic nature of the turbulent flow field. From chaos theory [7] it is known that the solution of certain nonlinear partial differential equations are sensitive to perturbations to initial conditions or parameters. The solutions of the Navier-Stokes equations are believed to exhibit this property and the behaviour has been demonstrated numerically and experimentally [8]. The divergence to infinity of the adjoint field makes it unusable for computing sensitivities of the objective with respect to perturbations in input parameters.

This paper presents a possible solution to this problem by controlling the growth of the adjoint field. An energy analysis of the unsteady compressible Navier-Stokes adjoint equations reflects that a single term contributes to the growth of the energy of the adjoint field while another viscous-like term dissipates the adjoint energy. This suggests the idea that adding artificial viscosity to the adjoint equations can dampen the adjoint fields. The maximum singular value of the growth term matrix gives an indication of the regions in the flow where the adjoint term is diverging at an exponential rate. By the addition of minimal artificial viscosity in these regions the growth of the adjoint energy can be curbed and at the same time the accuracy of the derivatives obtained from the adjoint solutions can potentially be maintained.

\section{PROBLEM SETUP}

The turbomachinery problem of interest is transonic flow over a highly loaded turbine inlet guide vane designed by researchers at Von Karman Institude (VKI) [9], shown in Figure 1.

Subsonic flow enters from the inlet on the left side, accelerates as it goes around the suction side and reaches close to the speed of sound near the trailing edge of the vane. The boundary layer transitions from laminar to turbulent at the suction side near the trailing edge as shown in Figure 2. The point of transition is highly dependent on the turbulent intensity of the flow at the inlet. The flow then separates at the trailing edge producing a turbulent wake. Due to boundary layer development on the suction and pressure sides and mixing in the wake there is a significant loss in stagnation pressure of the fluid.

The Reynolds number for this setup is $10^{6}$. The isentropic Mach number is 0.9. The vane has a chord length of 67.647 $\mathrm{mm}$ and is at angle of $\gamma=55$ degrees to the axial direction which is the same as the inlet flow direction. The vanes are in a linear cascade and the pitch is 0.85 times the chord length. 


\section{Leading edge

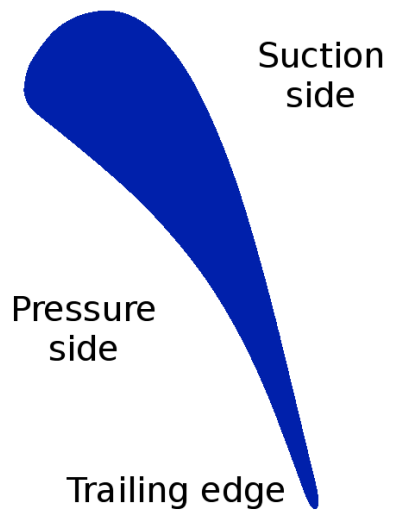

FIGURE 1. TURBINE VANE GEOMETRY

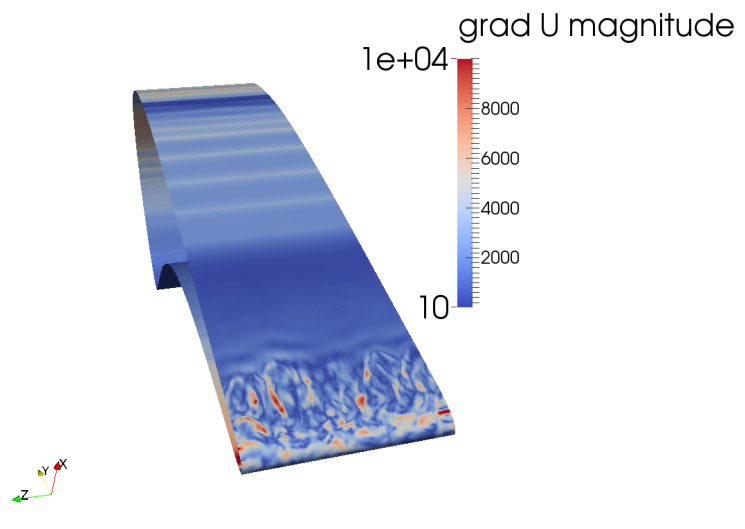

FIGURE 2. CONTOUR PLOT OF SHEAR STRESS ON THE SURFACE OF THE VANE. NON-DIMENSIONALIZED WITH RESPECT TO FLOW VELOCITY MACH 0.9. REYNOLDS NUMBER 1 MILLION, TURBULENT INTENSITY $1 \%$

In the simulation, periodic boundary conditions are imposed on the top and bottom. The spanwise extent of the numerical setup is restricted to $10 \mathrm{~mm}$. Numerical studies of this problem have shown that this is sufficient to capture the dynamics of turbulence [1]. The vane surface is assumed to be isothermal.

The design objective for this problem is an infinite timeaveraged and mass flow averaged stagnation pressure loss coefficient $\left(\bar{p}_{l}\right) 16 \mathrm{~mm}$ downstream of the vane on a surface parallel to the inlet plane. As mentioned before due to boundary layer development and mixing in the wake downstream of the vane there is a large drop in the stagnation pressure which leads to loss in performance. Hence, there is an interest in minimizing the pressure loss. In practice, the time average for the objective is per-

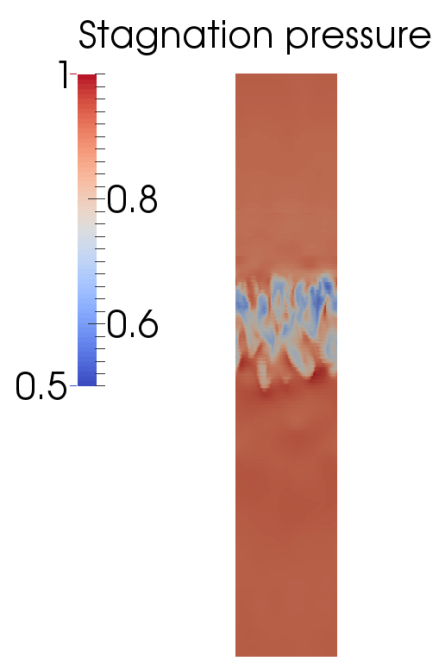

FIGURE 3. STAGNATION PRESSURE ON A VERTICAL SPANWISE CROSS SECTION 10 MM DOWNSTREAM OF THE TRAILING EDGE OF THE VANE. NON-DIMENSIONALIZED WITH RESPECT TO INLET STAGNATION PRESSURE

formed for an interval which is sufficient to provide a reasonably accurate estimate of the infinite time average. In this problem it is equal to the time it takes for the flow to pass from the inlet to the outlet which comes to be approximately $2 \mathrm{~ms}$. Though the interval length might seem small, it encompasses several time scales of the turbulence in the wake. The formula for $\bar{p}_{l}$ is

$$
\begin{aligned}
\bar{p}_{l} & =\frac{\bar{p}_{t, l}}{p_{t, i n}} \\
\bar{p}_{t, l} & =\lim _{T \rightarrow \infty} \frac{1}{T} \int_{0}^{T} \frac{\int_{S} \rho_{p} u_{n}\left(p_{t, i n}-p_{t, p}\right) d S}{\int_{S} \rho_{p} u_{n} d S} d t \\
p_{t, p} & =p_{p}\left(1+\frac{\gamma-1}{2} M_{p}^{2}\right)^{\frac{\gamma}{\gamma-1}}
\end{aligned}
$$

\section{Time averaged objectives}

Observations of turbulent flows indicate that statistics of turbulence like time-averaged mean of pressure loss coefficient are well-defined, stable quantities [10,11]. Other than relatively rare cases that exhibit flow hysterisis, these statistics are insensitive to initial conditions. In dynamical systems theory, an autonomous system is called ergodic if infinite time averages are independent of initial condition. An infinite time average of such ergodic systems is proven to be differentiable to parameters of the system under additional assumptions $[12,13]$. This theory is consistent with observations in turbulent flows, in which the statistics are 


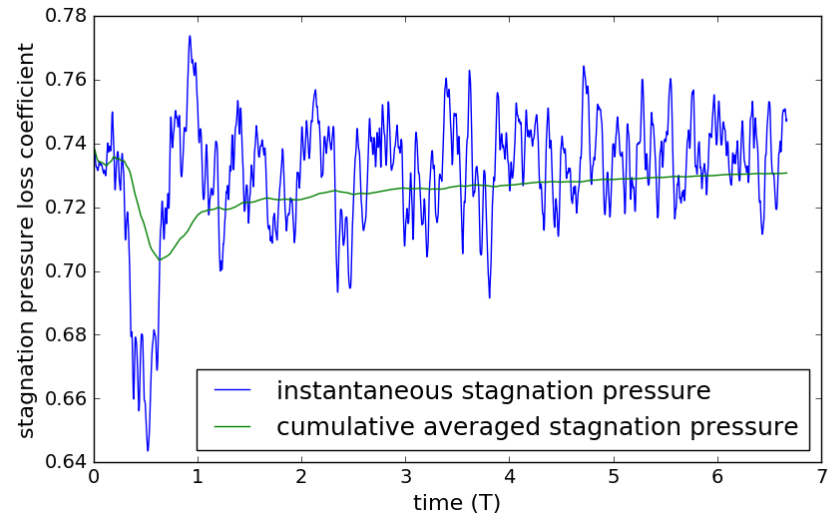

FIGURE 4. INSTANTANEOUS AND TIME-AVERAGED STAGNATION PRESSURE LOSS COEFFICEINT FOR THE VANE. XAXIS DENOTES THE TIME NORMALIZED BY THE TIME IT TAKES FOR THE FLOW TO PASS FROM THE INLET TO OUTLET

found to depend continuously on parameters when the flow is away from bifurcations [14]. Hence, we assume that the pressure loss coefficient is smooth as a function of inputs like source term perturbations to the compressible Navier-Stokes equations and shape parameters, or in other words at least the first derivative exists. In this particular problem the averaging is started after simulating the system to a statistically stationary state, which took about 5 flow throughs starting from a uniform zero velocity initial condition. Figure 4 shows the convergence of the pressure loss coefficient objective as the averaging interval is increased.

\section{Physics}

The problem can be physically modelled using the compressible Navier-Stokes equations with the ideal gas law as an approximation for the state equation and appropriate inlet, outlet and wall boundary conditions.

In $\mathbf{x} \in D, t \in[0, T]$,

$$
\begin{aligned}
& \frac{\partial \rho}{\partial t}+\nabla \cdot(\rho \mathbf{u})=0 \\
& \frac{\partial(\rho \mathbf{u})}{\partial t}+\nabla \cdot(\rho \mathbf{u u})+\nabla p=\nabla \cdot \sigma \\
& \frac{\partial(\rho E)}{\partial t}+\nabla \cdot(\rho E \mathbf{u}+p \mathbf{u})=\nabla \cdot(\mathbf{u} \cdot \sigma+\alpha \nabla e) \\
& \sigma=\mu\left(\nabla \mathbf{u}+\nabla \mathbf{u}^{T}\right)-\frac{2 \mu}{3}(\nabla \cdot \mathbf{u}) \mathbf{I} \\
& p=(\gamma-1) \rho e \\
& e=E-\frac{\mathbf{u} \cdot \mathbf{u}}{2}
\end{aligned}
$$

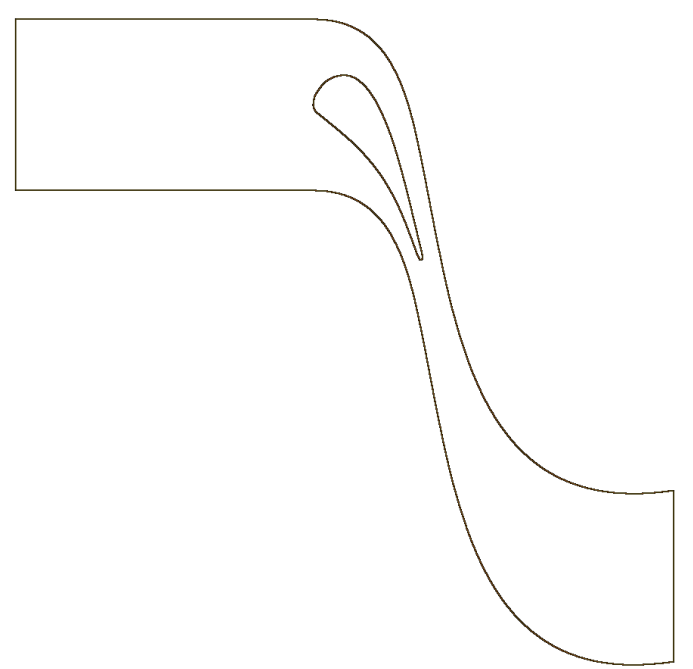

FIGURE 5. TURBINE VANE COMPUTATIONAL DOMAIN

For simulating turbulence, LES provides a way for resolving the large scale features of the flow and modelling the small scale structures. Typically when performing an LES a sub-grid scale (SGS) model like Smagorinsky or Vreman [15] is used, but as this problem is simulated using a second order finite volume method no SGS model is used. This approach in literature known as Implicit LES (ILES). It has been shown that when using a lower order method the numerical viscosity from the grid might be of the same order as the subgrid scale viscosity [16]. In the case of 2-D the simulation is just based off the unsteady compressible Navier-Stokes equation.

\section{Numerical methods}

The flow solver is written in Python with the help of numpy and scipy libraries and discretizes the equations over general unstructured meshes. For time marching a strong stability preserving third order Runge-Kutta method [17] is used. An approximate Roe [18] solver is employed for propagating shocks and discontinuities. Non reflecting boundary conditions are used for the inlet and outlet. Parallelization is accomplished using the Message Passing Interface (MPI) library.

The computational domain of the problem is shown in Figure 5. The simulations are performed on a 2-D and 3-D version of the problem. The 3-D mesh is just the 2-D mesh extended to 3-D and discretized uniformly in the spanwise direction. The mesh is a hybrid structured/unstructured mesh. It is structured in the inlet, outlet regions and around the surface of the vane and is unstructured in the remaining areas. The smallest cell size is maintained below $0.5 \mathrm{~mm}$ in regions away from the wall. This corresponds to a ratio of 4 between the Kolmogorov length scale and cell size at the inlet of the flow. To capture all the significant eddies of the flow (such an LES is called a wall resolved 
LES) near the wall, the wall normal cell width has to be below 1 in terms of wall units [19]. This puts a constraint on the time step to be of the order of a few nanoseconds, tremendously increasing the simulation cost. To be able to run the simulation in a reasonable time frame the maximum $y+$ is kept at $10, z+$ at 25 , and $x+$ at 150 . This results in an under resolved LES. In the future, a wall model might help alleviate this problem by allowing the mesh to have a higher $y+$. For now, the simulations are run without any wall model to get some initial unsteady adjoint results.

\section{UNSTEADY ADJOINT}

The unsteady adjoint method provides a way for computing derivatives of an objective dependent on the state of a system with respect to input parameters where the state is constrained by a time-dependent partial differential equation. Rewriting Equation 4 in vector form

$$
\begin{aligned}
\frac{\partial \mathbf{w}}{\partial t}+\nabla \cdot \mathbf{F}=\nabla \cdot \mathbf{F}^{\mathbf{v}} \\
\mathbf{w}=\left(\begin{array}{c}
\rho \\
\rho \mathbf{u} \\
\rho E
\end{array}\right) \\
\mathbf{F}=\left(\begin{array}{c}
\rho \mathbf{u} \\
\rho \mathbf{u u} \\
(\rho E+p) \mathbf{u}
\end{array}\right) \\
\mathbf{F}^{\mathbf{v}}=\left(\begin{array}{c}
0 \\
\sigma \\
\mathbf{u} \cdot \sigma+\alpha \nabla e
\end{array}\right)
\end{aligned}
$$

Using the Einstein summation notation in the Euclidean space Equation 5 can be simplified to (with the addition of a source term)

$$
\frac{\partial w_{i}}{\partial t}+\frac{\partial F_{i j}}{\partial x_{j}}=\frac{\partial F_{i j}^{v}}{\partial x_{j}}+s_{i}, i=1 \ldots .5
$$

Consider a time-averaged objective on the boundary surface $(S)$, $T$ is a large enough time to estimate the infinite time average with required accuracy. $J$ includes the factor $\frac{1}{T}$.

$$
\bar{J}=\int_{0}^{T} \int_{S} J\left(w_{i}\right) d S d t
$$

The first step in deriving the adjoint equations is to linearize the governing equation and form the Lagrangian,

$$
\begin{aligned}
\delta \bar{J} & =\int_{0}^{T} \int_{S}\left(\frac{\partial J}{\partial w_{i}} \delta w_{i}\right) d S d t \\
& +\int_{0}^{T} \int_{V} \hat{w}_{i}\left(\frac{\partial \delta w_{i}}{\partial t}+\frac{\partial \delta F_{i j}-\delta F_{i j}^{v}}{\partial x_{j}}-\delta s_{i}\right) d V d t
\end{aligned}
$$

Integrating the second term by parts in time and space,

$$
\begin{aligned}
\delta \bar{J} & =\int_{0}^{T} \int_{S}\left(\frac{\partial J}{\partial w_{i}} \delta w_{i}\right) d S d t+\int_{V}\left(\hat{w}_{i \mid T} \delta w_{i \mid T}-\hat{w}_{i \mid 0} \delta w_{i \mid 0}\right) d V \\
& -\int_{0}^{T} \int_{V} \frac{\partial \hat{w}_{i}}{\partial t} \delta w_{i} d V d t+\int_{0}^{T} \int_{S} \hat{w}_{i}\left(\delta F_{i j}-\delta F_{i j}^{v}\right) n_{j} d S d t \\
& -\int_{0}^{T} \int_{V} \frac{\partial \hat{w}_{i}}{\partial x_{j}}\left(\delta F_{i j}-\delta F_{i j}^{v}\right) d V d t \\
& -\int_{0}^{T} \int_{V} \hat{w}_{i} \delta s_{i} d V d t
\end{aligned}
$$

Differentiating $F_{i j}, F_{i j}^{v}$ with respect to $w_{k}, F_{i j}^{v}$ with respect to $\frac{\partial w_{k}}{\partial x_{l}}$,

$$
A_{i j k}=\frac{\partial F_{i j}}{\partial w_{k}}, \quad A_{i j k}^{v}=\frac{\partial F_{i j}^{v}}{\partial w_{k}}, \quad D_{i j k l}=\frac{\partial F_{i j}^{v}}{\partial \frac{\partial w_{k}}{\partial x_{l}}}
$$

The terms can be rearranged to form,

$$
\begin{aligned}
\delta \bar{J}= & \int_{0}^{T} \int_{S}\left[\left(\frac{\partial J}{\partial w_{i}}+\hat{w}_{k}\left(A_{k j i}-A_{k j i}^{v}\right) n_{j}\right) \delta w_{i}-\hat{w}_{i} D_{i j k l} \delta \frac{\partial w_{k}}{\partial x_{l}} n_{j}\right] d S d t \\
& +\int_{V}\left(\hat{w}_{i \mid T} \delta w_{i \mid T}-\hat{w}_{i \mid 0} \delta w_{i \mid 0}\right) d V \\
& -\int_{0}^{T} \int_{V}\left(\frac{\partial \hat{w}_{i}}{\partial t}+\frac{\partial \hat{w}_{k}}{\partial x_{j}}\left(A_{k j i}-A_{k j i}^{v}\right)\right) \delta w_{i} d V d t \\
& +\int_{0}^{T} \int_{V} \frac{\partial \hat{w}_{i}}{\partial x_{j}} D_{i j k l} \frac{\partial \delta w_{k}}{\partial x_{l}} d V d t \\
& -\int_{0}^{T} \int_{V} \hat{w}_{i} \delta s_{i} d V d t
\end{aligned}
$$

The last term in Equation 11 can again be integrated by parts to form

$$
\begin{aligned}
\int_{0}^{T} \int_{V} \frac{\partial \hat{w}_{i}}{\partial x_{j}} D_{i j k l} \frac{\partial \delta w_{k}}{\partial x_{l}} d V d t & =\int_{0}^{T} \int_{S} \frac{\partial \hat{w}_{i}}{\partial x_{j}} D_{i j k l} \delta w_{k} n_{l} d S d t \\
& -\int_{0}^{T} \int_{V} \frac{\partial}{\partial x_{l}}\left(\frac{\partial \hat{w}_{i}}{\partial x_{j}} D_{i j k l}\right) \delta w_{k} d V d t
\end{aligned}
$$


Using no perturbation in the initial condition, $\delta w_{i \mid 0}=0$, the adjoint equation comes out to be

$$
-\frac{\partial \hat{w}_{i}}{\partial t}-\left(A_{k j i}-A_{k j i}^{v}\right) \frac{\partial \hat{w}_{k}}{\partial x_{j}}=\frac{\partial}{\partial x_{l}}\left(D_{k j i l} \frac{\partial \hat{w}_{k}}{\partial x_{j}}\right)
$$

with boundary condition on the surface

$$
\left(\frac{\partial J}{\partial w_{i}}+\hat{w}_{k}\left(A_{k j i}-A_{k j i}^{v}\right) n_{j}+\frac{\partial \hat{w}_{k}}{\partial x_{j}} D_{k j i l} n_{l}\right) \delta w_{i}-\hat{w}_{i} D_{i j k l} \delta \frac{\partial w_{k}}{\partial x_{l}} n_{j}=0
$$

And terminal condition $\hat{w}_{i, T}=0$. Notice that there is a terminal condition which implies that the adjoint equations have to be integrated backwards in time. The procedure to compute sensitivity of an objective for a set of perturbations involves first solving the compressible Navier-Stokes (primal) equations from time $t=0$ to $T$. After this, the adjoint equation is solved backwards in time from $t=T$ to 0 . The adjoint equation requires the solution of the primal equation at every time $t$. The sensitivity due to a perturbation in the source term can be obtained using,

$$
\delta \bar{J}=-\int_{0}^{T} \int_{V} \hat{w}_{i} \delta s_{i} d V d t
$$

\section{Implementation}

In practice, the adjoint equation is implemented as a discrete unsteady adjoint instead of the continuous unsteady adjoint as just derived in this section. The discrete adjoint has the advantage that it provides a derivative or adjoint sensitivities that are precise to machine precision when compared with finite difference sensitivities. The discrete adjoint is derived with the help of automatic differentiation provided by the Python package Theano [20,21]. Additionally, the checkpointing method is used to provide the adjoint equation with the necessary primal solutions at each point in time. This method overcomes the need for storing all the primal solutions (at every time step) in memory by saving snapshots of the primal solution in periodic intervals on disk. When the adjoint is simulated, the primal solutions in an interval are obtained by performing the primal simulation again for the specific interval.

\section{Results}

The unsteady adjoint method is tested on the turbine vane problem using an aerothermal objective. A total of 4 simulations are performed, a short time interval and a long time interval simulation on each of the 2-D and 3-D turbine vane problems. Figure 6 shows a contour plot of the density adjoint field for a cross section of the 3-D turbine vane. The adjoint magnitude is large in the trailing edge region and the leading edge. The flow

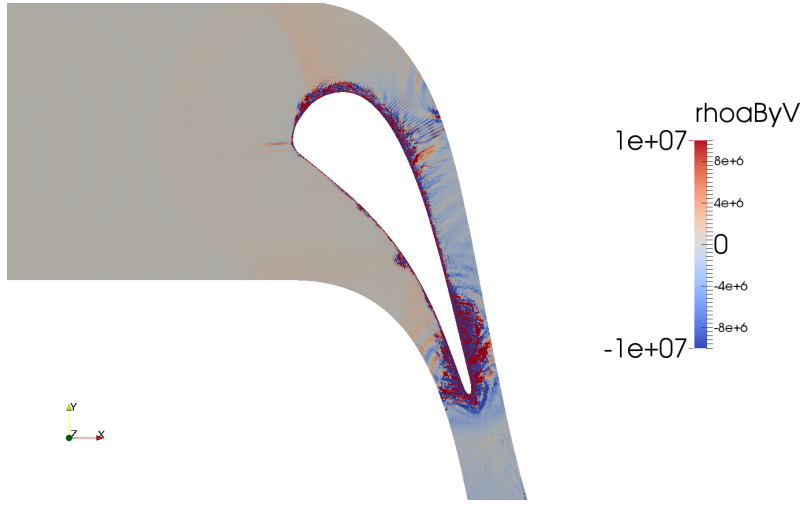

FIGURE 6. A VISUALIZATION OF THE DENSITY ADJOINT FIELD FROM HALFWAY THROUGH A SHORT TIME 3-D UNSTEADY ADJOINT SIMULATION

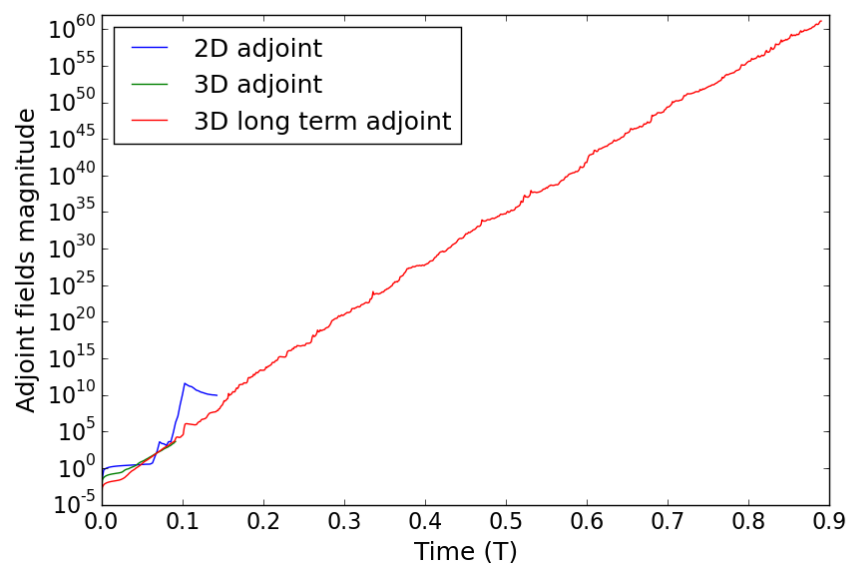

FIGURE 7. GROWTH OF ENERGY NORM OF ADJOINT FIELDS FOR DIFFERENT SIMULATIONS. $Y$-AXIS SHOWS ENERGY NORM OF A DIMENSIONAL CONSERVATIVE ADJOINT FIELD

is sensitive to perturbations in this area leading to the high values of the adjoint flow fields.

The 2-D and 3-D long time interval unsteady adjoints for the turbine vane diverge exponentially when simulated backwards in time. The actual values of the magnitude of the adjoint fields in Figure 7 are not important, but what is interesting to note is the growth rate of the adjoint fields. The 2-D adjoint diverges at a faster rate than the 3-D adjoint. This is because the $3^{\text {rd }}$ dimension provides a way to dissipate the growth in the adjoint flow field. The long time interval 3-D unsteady adjoint shows exponential growth throughout the length of the simulation and it's $L_{2}$ norm reaches very high magnitudes of around $10^{60}$.

The high magnitudes of the adjoint fields make the adjoint 
TABLE 1. COMPARISON BETWEEN ADJOINT AND FINITE DIFFERENCE SENSITIVITIES FOR A HEAT TRANSFER OBJECTIVE

\begin{tabular}{|c|l|l|l|}
\hline Simulation & $\mathrm{T}$ & Finite Diff. & Adjoint \\
\hline 2-D & 0.01 & $4.340 \mathrm{e}-5$ & $4.341 \mathrm{e}-5$ \\
2-D & 0.1 & 0.00695 & 640041 \\
3-D & 0.1 & 0.005904 & 0.005923 \\
3-D & 1 & 0.00710 & $\sim 10^{34}$ \\
\hline
\end{tabular}

sensitivities worthless in the case of the long time interval 2-D unsteady adjoint $(T=0.1)$ and 3 -D unsteady adjoint $(T=1)$ simulations, where $T$ is the simulation time normalized with respect to a single flow through time. Table 1 gives the actual values of the sensitivities of the aerothermal objective with respect to Gaussian shaped source term perturbations in the conservative flow fields upstream of the vane. The short time interval 2-D and 3 -D unsteady adjoint simulations provide the correct sensitivities. This is because the $L_{2}$ norm of the adjoint fields haven't reached a large enough magnitude for most of the simulation time. This result shows that for an objective which only requires a short time average, the unsteady adjoint method can potentially provide the correct gradient.

\section{STABILIZING ADJOINT EQUATIONS}

Due to the chaotic nature of a turbulent fluid flow, the $L_{2}$ norm of an unsteady adjoint solution diverges exponentially when the adjoint equations are solved backwards in time. This is primarily due to the sensitivity of the solution field with respect to small perturbations in the initial flow field or parameters of the fluid system.

$$
\|\hat{w}\|_{L_{2}(D)}=\left(\int_{V} \hat{w}_{i} \hat{w}_{i} d V\right)^{\frac{1}{2}}
$$

Wang [5] performed an energy norm analysis of the adjoint equations for a fluid flow governed by the incompressible NavierStokes equations and found out that there are two terms which govern the change in adjoint energy of the system. The first is a growth term which is large in regions where the matrix norm of $\nabla \mathbf{u}$ is large, meaning that regions having a large gradients in velocity contribute to the divergence of adjoint energy. The second is a dissipation term which tries to reduce the adjoint energy and is scaled by the viscosity of the fluid. The adjoint energy diverges to infinity when the growth term dominates the dissipation term. This analysis shows that if additional viscosity is added to the adjoint equations the dissipation term can limit the growth of the adjoint field.
Blonigan [6] performed numerical experiments of adding uniform artificial viscosity to the adjoint equations and was successful in inhibiting the exponential growth of the adjoint field. But this also resulted in the corruption of the sensitivities obtained from the adjoint solution. A potential fix to the latter problem is to add viscosity in certain regions of the fluid flow to only the adjoint equations where the adjoint field has a high rate of growth. This idea is explored by applying the energy analysis method on the unsteady adjoint of the compressible NavierStokes equations.

\section{Symmetrization}

Performing the energy analysis on the adjoint of the symmetrized Navier-Stokes equations comes out to be more useful than on the conservative Navier Stokes equations. It can be shown that if the adjoint energy norm of the symmetrized equations is bounded then the adjoint energy of the conservative equations is also bounded. Hence from here forward the focus will be on the adjoint of the symmetrized equations.

Symmetrization of the Navier-Stokes equations means making the tensors $A_{i j k}$ and $D_{i j k l}$ symmetric in $i$ and $k$. The analysis is performed on the Euler equations, but the symmetrization procedure works out for the compressible Navier-Stokes equations as demonstrated in [22]. Using the quasi-linear form of the Euler equation,

$$
\frac{\partial w_{i}}{\partial t}+A_{i j k} \frac{\partial w_{k}}{\partial x_{j}}=0
$$

Symmetrizing by a transformation of the conservative variables to symmetrized variables, $\delta v_{i}=T_{i k} \delta w_{k}$,

$$
T_{i k}^{-1} \frac{\partial v_{k}}{\partial t}+A_{i j k} T_{k m}^{-1} \frac{\partial v_{m}}{\partial x_{j}}=0
$$

Pre-multiplying by $T_{l i}$

$$
\frac{\partial v_{l}}{\partial t}+T_{l i} A_{i j k} T_{k m}^{-1} \frac{\partial v_{m}}{\partial x_{j}}=0
$$

$T$ can be chosen such that $\hat{A}_{l j m}=T_{l i} A_{i j k} T_{k m}^{-1}$ is symmetric giving the symmetrized Euler equations,

$$
\frac{\partial v_{l}}{\partial t}+\hat{A}_{l j m} \frac{\partial v_{m}}{\partial x_{j}}=0
$$

The symmetrizer from primitive to symmetric variables, $\delta v_{i}=$ 
$S_{i k} \delta q_{i}$ is,

$$
S=\left(\begin{array}{ccccc}
\frac{c}{\sqrt{\gamma \rho}} & 0 & 0 & 0 & 0 \\
0 & 1 & 0 & 0 & 0 \\
0 & 0 & 1 & 0 & 0 \\
0 & 0 & 0 & 1 & 0 \\
-\frac{c}{\rho \sqrt{\gamma(\gamma-1)}} & 0 & 0 & 0 & \sqrt{\frac{\gamma}{\gamma-1}} \frac{1}{\rho c}
\end{array}\right)
$$

The transformation from conservative to primitive variables is $\delta q_{i}=V_{i k} \delta w_{i}$

$$
V=\left(\begin{array}{ccccc}
1 & 0 & 0 & 0 & 0 \\
\frac{u_{1}}{\rho} & \frac{1}{\rho} & 0 & 0 & 0 \\
\frac{u_{2}}{\rho} & 0 & \frac{1}{\rho} & 0 & 0 \\
\frac{u_{3}}{\rho} & 0 & 0 & \frac{1}{\rho} & 0 \\
\frac{(\gamma-1) u_{i} u_{i}}{2}-(\gamma-1) u_{1}-(\gamma-1) u_{2}-(\gamma-1) u_{3} & (\gamma-1)
\end{array}\right)
$$

With $T_{i k}=S_{i j} V_{j k}$.

$$
\hat{A}_{i j k} \text { is given by }
$$

$$
\begin{aligned}
& \hat{A}_{: 1:}=\left(\begin{array}{ccccc}
u_{1} & \frac{c}{\sqrt{\gamma}} & 0 & 0 & 0 \\
\frac{c}{\sqrt{\gamma}} & u_{1} & 0 & 0 & \sqrt{\frac{\gamma-1}{\gamma}} c \\
0 & 0 & u_{1} & 0 & 0 \\
0 & 0 & 0 & u_{1} & 0 \\
0 & \sqrt{\frac{\gamma-1}{\gamma}} c & 0 & 0 & u_{1}
\end{array}\right) \\
& \hat{A}_{: 2:}=\left(\begin{array}{ccccc}
u_{2} & 0 & \frac{c}{\sqrt{\gamma}} & 0 & 0 \\
0 & u_{2} & 0 & 0 & 0 \\
\frac{c}{\sqrt{\gamma}} & 0 & u_{2} & 0 & \sqrt{\frac{\gamma-1}{\gamma}} c \\
0 & 0 & 0 & u_{2} & 0 \\
0 & 0 & \sqrt{\frac{\gamma-1}{\gamma}} c & 0 & u_{2}
\end{array}\right) \\
& \hat{A}_{: 3:}=\left(\begin{array}{ccccc}
u_{3} & 0 & 0 & \frac{c}{\sqrt{\gamma}} & 0 \\
0 & u_{3} & 0 & 0 & 0 \\
0 & 0 & u_{3} & 0 & 0 \\
\frac{c}{\sqrt{\gamma}} & 0 & 0 & u_{3} & \sqrt{\frac{\gamma-1}{\gamma}} c \\
0 & 0 & 0 & \sqrt{\frac{\gamma-1}{\gamma}} c & u_{3}
\end{array}\right)
\end{aligned}
$$

The adjoint equation for the symmetrized equation comes out to be slightly different as $\hat{A}_{l j m}$ is not a Jacobian of the flux term of the symmetric variables.

$$
\begin{aligned}
\delta \bar{J} & =\int_{0}^{T} \int_{S}\left(\frac{\partial J}{\partial v_{i}} \delta v_{i}\right) d S d t \\
& +\int_{0}^{T} \int_{V} \hat{v}_{i}\left(\frac{\partial \delta v_{i}}{\partial t}+\hat{A}_{i j k} \frac{\partial \delta v_{k}}{\partial x_{j}}+\delta \hat{A}_{i j k} \frac{\partial v_{k}}{\partial x_{j}}\right) d V d t
\end{aligned}
$$

Linearizing $\hat{A}_{i j k}$ using $B_{i j k l}=\frac{\partial \hat{A}_{i j k}}{\partial v_{l}}$ and integrating by parts in time and space,

$$
\begin{aligned}
\delta \bar{J}= & \int_{0}^{T} \int_{S}\left(\frac{\partial J}{\partial v_{i}} \delta v_{i}\right) d S d t \\
& +\int_{V}\left(\hat{v}_{i \mid T} \delta v_{i \mid T}-\hat{v}_{i \mid 0} \delta v_{i \mid 0}\right) d V-\int_{0}^{T} \int_{V} \frac{\partial \hat{v}_{i}}{\partial t} \delta v_{i} d V d t \\
& +\int_{0}^{T} \int_{S} \hat{v}_{i} \hat{A}_{i j k} \delta v_{k} n_{j} d S-\int_{0}^{T} \int_{V} \frac{\partial\left(\hat{v}_{i} \hat{A}_{i j k}\right)}{\partial x_{j}} \delta v_{k} d V d t \\
& +\int_{0}^{T} \int_{V} \hat{v}_{i} B_{i j k l} \delta v_{l} \frac{\partial v_{k}}{\partial x_{j}} d V d t
\end{aligned}
$$

giving rise to the adjoint equation,

$$
-\frac{\partial \hat{v}_{i}}{\partial t}-\hat{A}_{k j i} \frac{\partial \hat{v}_{k}}{\partial x_{j}}-\left(B_{k j i l}-B_{k j l i}\right) \frac{\partial v_{l}}{\partial x_{j}} \hat{v}_{k}=0
$$

The viscous term follows the same derivation process as done for the conservative adjoint in the previous section with one important difference, the $\mathbf{F}^{\mathbf{v}}$ term is considered to be purely a function of $\nabla \mathbf{v}$ on the assumption that $\frac{\mu}{\rho}$ and $\frac{\alpha}{\rho}$ are constant for linearization purposes. This is also known as the frozen viscosity assumption and implies that the $A_{k j i}^{v}$ term can be ignored. The $\hat{D}_{i j k l}$ tensor comes out to be

$$
\begin{aligned}
& \hat{D}_{: 1: 1}=\left(\begin{array}{ccccc}
0 & 0 & 0 & 0 & 0 \\
0 & \frac{4}{3} \frac{\mu}{\rho} & 0 & 0 & 0 \\
0 & 0 & \frac{\mu}{\rho} & 0 & 0 \\
0 & 0 & 0 & \frac{\mu}{\rho} & 0 \\
0 & 0 & 0 & 0 & \frac{\gamma \alpha}{\rho}
\end{array}\right)
\end{aligned}
$$

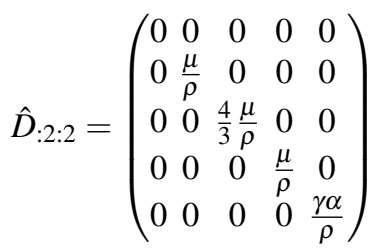




$$
\begin{gathered}
\hat{D}_{: 3: 3}=\left(\begin{array}{ccccc}
0 & 0 & 0 & 0 & 0 \\
0 & \frac{\mu}{\rho} & 0 & 0 & 0 \\
0 & 0 & \frac{\mu}{\rho} & 0 & 0 \\
0 & 0 & 0 & \frac{4}{3} \frac{\mu}{\rho} & 0 \\
0 & 0 & 0 & 0 & \frac{\gamma \alpha}{\rho}
\end{array}\right) \\
\hat{D}_{: 1: 2}=\hat{D}_{: 2: 1}=\frac{1}{2}\left(\begin{array}{cccccc}
0 & 0 & 0 & 0 & 0 \\
0 & 0 & \frac{1}{3} \frac{\mu}{\rho} & 0 & 0 \\
0 & \frac{1}{3} & \frac{\mu}{\rho} & 0 & 0 & 0 \\
0 & 0 & 0 & 0 & 0 \\
0 & 0 & 0 & 0 & 0
\end{array}\right) \\
\hat{D}_{: 1: 3}=\hat{D}_{: 3: 1}=\frac{1}{2}\left(\begin{array}{cccccc}
0 & 0 & 0 & 0 & 0 \\
0 & 0 & 0 & \frac{1}{3} \frac{\mu}{\rho} & 0 \\
0 & 0 & 0 & 0 & 0 \\
0 & \frac{1}{3} \frac{\mu}{\rho} & 0 & 0 & 0 \\
0 & 0 & 0 & 0 & 0
\end{array}\right) \\
\hat{D}_{: 2: 3}=\hat{D}_{: 3: 2}=\frac{1}{2}\left(\begin{array}{cccccc}
0 & 0 & 0 & 0 & 0 \\
0 & 0 & 0 & 0 & 0 \\
0 & 0 & 0 & \frac{1}{3} \frac{\mu}{\rho} & 0 \\
0 & 0 & \frac{1}{3} \frac{\mu}{\rho} & 0 & 0 \\
0 & 0 & 0 & 0 & 0
\end{array}\right)
\end{gathered}
$$

As discussed before, if the conservative adjoint field at any point of time is bounded then the symmetrized adjoint field is bounded and the vice versa is also true. This can be shown in the following way: the sensitivity due to a perturbation can be computed from either the conservative adjoint solution or symmetrized adjoint solution.

$$
\begin{aligned}
\delta \bar{J} & =-\int_{0}^{T} \int_{V} \hat{w}_{i} \delta s_{i} d V d t \\
& =-\int_{0}^{T} \int_{V} \hat{v}_{k} \delta s_{k}^{v} d V d t=-\int_{0}^{T} \int_{V} \hat{v}_{k} T_{k i} \delta s_{i} d V d t
\end{aligned}
$$

So,

$$
\begin{aligned}
\hat{w}_{i} & =T_{k i} \hat{v}_{i} \\
\|\hat{\mathbf{w}}\|_{L_{2}(D)} & \leq\left\|\mathbf{T}^{T}\right\|_{L_{2}(D)}\|\hat{\mathbf{v}}\|_{L_{2}(D)}
\end{aligned}
$$

The transformation matrix consists of bounded component fields and hence it's matrix $L_{2}$ norm is bounded. This implies that if the symmetrized adjoint is bounded then the conservative adjoint is also bounded.

\section{Energy Analysis}

To study how the adjoint diverges the time derivative of the adjoint energy $E_{\hat{v}}=\|\hat{v}\|_{L_{2}(D)}$ is analyzed. The adjoint energy is basically the sum of the squares of the component-wise $L_{2}$ norms. The norms can be summed without dimensional scaling as the components of the symmetrized adjoint field have the same dimensions because of the fact that all the symmetrized NavierStokes variables have the same dimensions.

$$
-\frac{1}{2} \frac{d E_{\hat{v}}}{d t}=-\frac{1}{2} \frac{\partial}{\partial t}\left(\int_{V} \hat{v}_{i} \hat{v}_{i}\right) d V=-\int_{V} \hat{v}_{i} \frac{\partial \hat{v}_{i}}{\partial t} d V
$$

pre-multiplying the adjoint equation by $\hat{v}_{i}$ and integrating over the entire domain,

$$
\begin{aligned}
\frac{d E_{\hat{v}}}{d t} & =\int_{V} \hat{v}_{i}\left(\hat{A}_{k j i} \frac{\partial \hat{v}_{k}}{\partial x_{j}}+\left(B_{k j i l}-B_{k j l i}\right) \frac{\partial v_{l}}{\partial x_{j}} \hat{v}_{k}\right. \\
& \left.+\frac{\partial}{\partial x_{l}}\left(\hat{D}_{k j i l} \frac{\partial \hat{v_{k}}}{\partial x_{j}}\right)\right) d V
\end{aligned}
$$

using $B_{k j i l}=\frac{\partial \hat{A}_{k j i}}{\partial v_{l}}$ the first term can be rewritten as,

$$
\begin{aligned}
\int_{V} \hat{v}_{i} \hat{A}_{k j i} \frac{\partial \hat{v}_{k}}{\partial x_{j}} d V & =\int_{S} \hat{v}_{i} \hat{A}_{k j i} \hat{v}_{k} n_{j} d S \\
& -\int_{V} \frac{\partial \hat{v}_{i}}{\partial x_{j}} \hat{A}_{k j i} \hat{v}_{k} d V-\int_{V} \hat{v}_{i} B_{k j i l} \frac{\partial v_{l}}{\partial x_{j}} \hat{v}_{k} d V
\end{aligned}
$$

Using symmetry of $\hat{A}_{k j i}$ in $i$ and $k$,

$$
\int_{V} \hat{v}_{i} \hat{A}_{k j i} \frac{\partial \hat{v}_{k}}{\partial x_{j}} d V=\frac{1}{2}\left(\int_{S} \hat{v}_{i} \hat{A}_{k j i} \hat{v}_{k} n_{j} d S-\int_{V} \hat{v}_{i} B_{k j i l} \frac{\partial v_{l}}{\partial x_{j}} \hat{v}_{k} d V\right)
$$

The second term in the energy equations is,

$$
\int_{V} \frac{\partial}{\partial x_{l}}\left(\hat{D}_{k j i l} \frac{\partial \hat{v}_{k}}{\partial x_{j}}\right) d V=\int_{S} \hat{v}_{i} \hat{D}_{k j i l} \frac{\partial \hat{v}_{k}}{\partial x_{j}} n_{l} d S-\int_{V} \frac{\partial \hat{v}_{i}}{\partial x_{l}} \hat{D}_{k j i l} \frac{\partial \hat{v}_{k}}{\partial x_{j}} d V
$$

So, the adjoint energy equation becomes,

$$
\begin{aligned}
\frac{d E_{\hat{v}}}{d t} & =\int_{V} \hat{v}_{i}\left[\left(\frac{B_{k j i l}}{2}-B_{k j l i}\right) \frac{\partial v_{l}}{\partial x_{j}}\right] \hat{v}_{k} d V-\int_{V} \frac{\partial \hat{v}_{i}}{\partial x_{l}} \hat{D}_{k j i l} \frac{\partial \hat{v}_{k}}{\partial x_{j}} d V \\
& +\int_{S} \hat{v}_{i}\left(\hat{D}_{k j i l}\right) \frac{\partial \hat{v}_{k}}{\partial x_{j}} n_{l} d S+\frac{1}{2} \int_{S} \hat{v}_{i} \hat{A}_{k j i} \hat{v}_{k} n_{j} d S
\end{aligned}
$$


Let,

$$
\begin{aligned}
& M_{1}=\frac{B_{k j i l}}{2} \frac{\partial v_{l}}{\partial x_{j}}=\frac{\partial \hat{A}_{k j i}}{\partial q_{l}} \frac{\partial q_{l}}{\partial x_{j}}, \\
& M_{2}=B_{k j l i} \frac{\partial v_{l}}{\partial x_{j}}=\frac{\partial \hat{A}_{k j l}}{\partial q_{m}} \frac{\partial q_{m}}{\partial v_{i}} \frac{\partial v_{l}}{\partial x_{j}}=\frac{\partial \hat{A}_{k j l}}{\partial q_{m}} S_{m i}^{-1} \frac{\partial v_{l}}{\partial x_{j}}
\end{aligned}
$$

The first volumetric term in 43 is a quadratic term in $\hat{\mathbf{v}}$ scaled by the matrix $M=M_{1}-M_{2}$. This is the term that primarily contributes to the diverging growth of the adjoint energy.

The second volumetric term in Equation 43 is the dissipation term. Simplification shows that it is proportional to $\left\|\nabla \hat{v}_{i}\right\|^{2}$ scaled by the viscous coefficient. As the sign in front of the term is negative, this term reduces the growth of the adjoint energy.

The boundary terms are quadratic and can potentially contribute to the growth of the adjoint energy. On the inlet and outlet of the domain, characteristic boundary conditions are applied as the fluid is practically inviscid on these boundaries. Doing so, the first boundary term in Equation 43 can be ignored. Denoting the characteristic Navier-Stokes variables by $z_{i}$ and the characteristic adjoint variables by $\hat{z}_{i}$, the boundary condition on the inlet and outlet can be written as,

$$
\hat{v}_{k} \hat{A}_{k j i} n_{j} \delta v_{i}=\hat{z}_{k} \Lambda_{l i} \delta z_{i}=0
$$

using the eigendecomposition of $\hat{A}_{k j i} n_{j}=Q_{k l} \Lambda_{l m} Q_{i m}$ and the identities $\delta v_{i}=Q_{k i} \delta z_{i}, \hat{z}_{i}=Q_{k i} \hat{v}_{k}$. On the inlet the characteristic variables coming into the domain are set. This corresponds to $\delta z_{i}=0$ for $i$ where $i^{t h}$ characteristic (or eigenvalues in the eigendecomposition) is negative. This in turn implies that $\hat{z}_{j}=0$ for $j$ where the $j^{t h}$ characteristic is positive, to ensure that the product is 0 . This means that $\hat{\mathbf{v}}$ belongs to the negative eigenspace of the matrix and so the second boundary term is always negative. Similarly, for the outlet, the outgoing characteristics are set, which corresponds to $\delta z_{i}=0$ for $i$ where $i^{\text {th }}$ characteristic is negative. Hence, for characteristic boundaries the second boundary term is always negative and it does not contribute to the growth of the adjoint. Wall boundaries require more analysis as in this case the viscous terms are important due to the presence of large gradients near the wall. For this paper, the walls are assumed to not contribute significantly to the growth of the adjoint term. This does not mean that the near wall regions adjoint fields do not contribute to the sensitivity, in fact as we shall see in the next section, they do.

The contribution from the objective source terms to the adjoint energy is linear and hence they don't directly contribute to the divergence of the adjoint fields.

$$
\begin{aligned}
\text { Setting } b=\frac{c}{\sqrt{\gamma}}, a & =\sqrt{\frac{\gamma-1}{\gamma}} c . \\
M_{1} & =\frac{1}{2}\left(\begin{array}{ccccc}
\nabla \cdot u & \nabla_{1} b & \nabla_{2} b & \nabla_{3} b & 0 \\
\nabla_{1} b & \nabla \cdot u & 0 & 0 & \nabla_{1} a \\
\nabla_{2} b & 0 & \nabla \cdot u & 0 & \nabla_{2} a \\
\nabla_{3} b & 0 & 0 & \nabla \cdot u & \nabla_{3} a \\
0 & \nabla_{1} a & \nabla_{2} a & \nabla_{3} a & \nabla \cdot u
\end{array}\right)
\end{aligned}
$$

$$
M_{2}=\left(\begin{array}{ccccc}
0 & \frac{b}{\rho} \nabla_{1} \rho & \frac{b}{\rho} \nabla_{2} \rho & \frac{b}{\rho} \nabla_{3} \rho & \frac{\sqrt{\gamma-1}}{2} \nabla \cdot u \\
0 & \nabla_{1} u_{1} & \nabla_{2} u_{1} & \nabla_{3} u_{1} & \frac{a}{2 p} \nabla_{1} p \\
0 & \nabla_{1} u_{2} & \nabla_{2} u_{2} & \nabla_{3} u_{2} & \frac{a}{2 p} \nabla_{2} p \\
0 & \nabla_{1} u_{3} & \nabla_{2} u_{3} & \nabla_{3} u_{3} & \frac{a}{2 p} \nabla_{3} p \\
0 & \frac{2}{\gamma-1} \nabla_{1} a & \frac{2}{\gamma-1} \nabla_{2} a & \frac{2}{\gamma-1} \nabla_{3} a & \frac{\gamma-1}{2} \nabla \cdot u
\end{array}\right)
$$

Analyzing the matrix $M$, which is the growth matrix, provides a way to find regions in the fluid flow where the adjoint is diverging. Using Cauchy-Schwartz inequality for matrix/vector norms

$$
\left|\hat{\mathbf{v}}^{T} \mathbf{M} \hat{\mathbf{v}}\right| \leq\|\mathbf{v}\|\|\mathbf{M v}\|
$$

Using the matrix induced 2-norm and the identity $\|\mathbf{M v}\|_{2} \leq$ $\sigma_{1}\|\mathbf{v}\|_{2}$, with $\sigma_{1}$ being the maximum singular value of $M$.

$$
\left|\hat{\mathbf{v}}^{T} \mathbf{M} \hat{\mathbf{v}}\right| \leq \sigma_{1}\|\hat{\mathbf{v}}\|_{2}^{2}
$$

The magnitude of $\sigma_{1}$ gives an indication of the regions where the adjoint energy growth is high. It has the dimensions $\frac{1}{T}$ and has the physical meaning of a rate of growth term.

Additional viscosity is added to the adjoint equations to curb the divergence of the adjoint field in the following form,

$$
-\frac{\partial \hat{w}_{i}}{\partial t}-\left(A_{k j i}-A_{k j i}^{v}\right) \frac{\partial \hat{w}_{k}}{\partial x_{j}}=\frac{\partial}{\partial x_{l}}\left(\left(D_{k j i l}+\lambda \sigma_{1} \delta_{k i} \delta_{j l}\right) \frac{\partial \hat{w}_{k}}{\partial x_{j}}\right)
$$

where $\lambda$ is a scaling factor that is problem specific and in this work is manually tuned. It has the dimensions of $L^{2}$ where $L$ denotes length. As an initial estimate the $\lambda$ can be set to the length scale of the chaotic flow that contributes to the divergence of the adjoint. For example, for the turbine case this is equal to the turbulent length scale in the wake. For 2-D chaotic flow over a cylinder it can be the length scale of the vortex shedding.

\section{Results}

The stabilized adjoint algorithm is tested on the 2-D turbine vane problem. The objective is the time-averaged and mass flow 


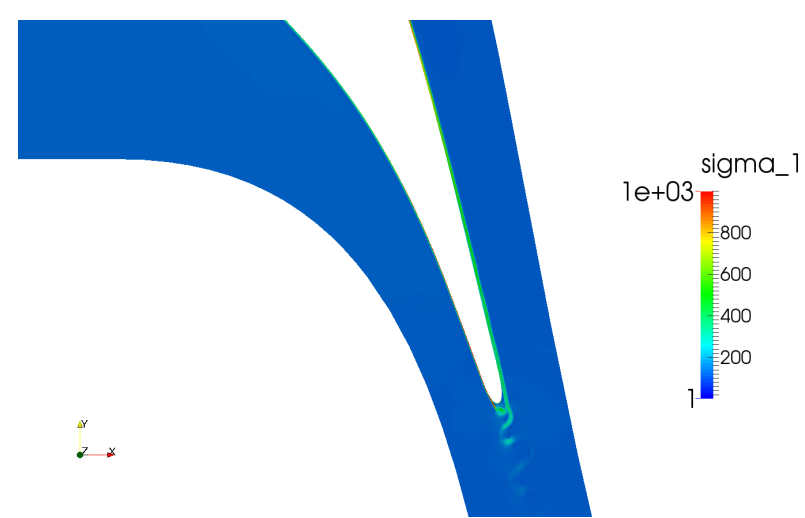

FIGURE 8. CONTOUR PLOT OF DIVERGENCE INDICATOR $\sigma_{1}$ FOR THE TURBINE VANE, NORMALIZED BY INVERSE OF A SINGLE FLOW THROUGH TIME

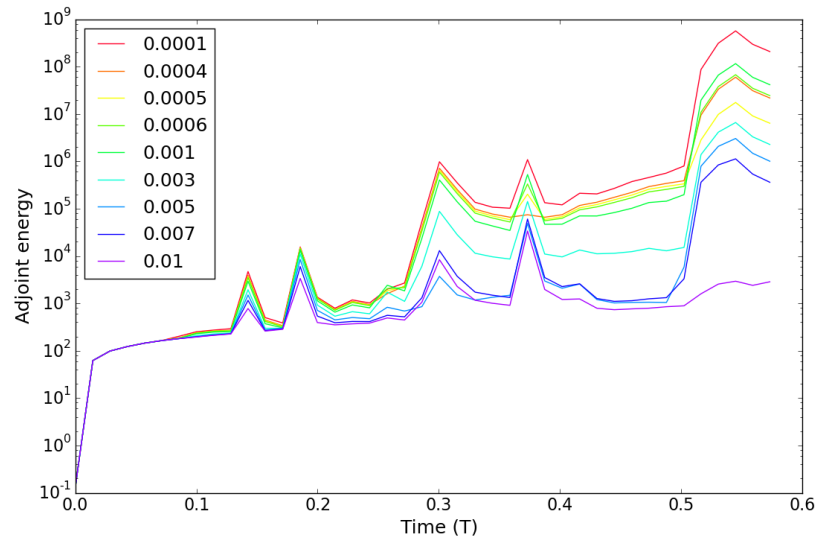

FIGURE 9. GROWTH OF ENERGY NORM OF ADJOINT FOR VARIOUS SCALING FACTORS. X-AXIS IS TIME NORMALIZED BY A SINGLE FLOW THROUGH TIME

averaged pressure loss coefficient given by Equation 1. Timeaveraging is performed over $1 / 10^{\text {th }}$ of a fluid flow through time.

Figure 8 shows the regions where the maximum singular value $\sigma_{1}$ of the matrix $M$ is large. As expected, the region in the boundary layer near the trailing edge is primarily responsible for the diverging adjoint and by adding additional dissipation in this region we can restrict the growth of the adjoint field.

Various values of $\lambda$ are tried from $\lambda=10^{-4}$ to $10^{-2}$. Figure 9 demonstrates the growth of energy norm of adjoint fields with time for the different values of $\lambda$. When the scaling factor is too low the additional viscosity does not change the adjoint solution by a significant amount and the $L_{2}$ norm of the adjoint fields stays high. On increasing the scaling factor the magnitude of the $L_{2}$ norm of the adjoint fields reduces, but still shows expo-

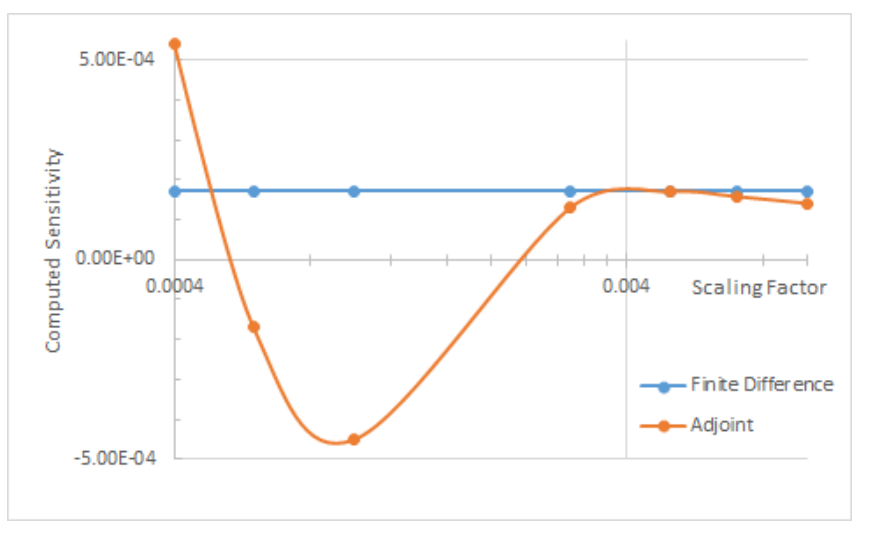

FIGURE 10. ERROR IN ADJOINT SENSITIVITY FOR VARIOUS SCALING FACTORS

\begin{tabular}{|c|l|c|}
\hline \multicolumn{3}{|c|}{ Sensitivities } \\
\hline Scaling factor $(\lambda)$ & Finite Difference & Adjoint \\
\hline 0.0001 & $1.71 \mathrm{e}-4$ & $3.44 \mathrm{e}-3$ \\
0.0004 & $1.71 \mathrm{e}-4$ & $5.42 \mathrm{e}-4$ \\
0.0006 & $1.71 \mathrm{e}-4$ & $-1.7 \mathrm{e}-4$ \\
0.001 & $1.71 \mathrm{e}-4$ & $-4.5 \mathrm{e}-4$ \\
0.003 & $1.71 \mathrm{e}-4$ & $1.30 \mathrm{e}-4$ \\
0.005 & $1.71 \mathrm{e}-4$ & $1.71 \mathrm{e}-4$ \\
0.007 & $1.71 \mathrm{e}-4$ & $1.59 \mathrm{e}-4$ \\
0.01 & $1.71 \mathrm{e}-4$ & $1.42 \mathrm{e}-4$ \\
\hline
\end{tabular}

TABLE 2. COMPARISON OF ADJOINT AND FINITE DIFFERENCE SENSITIVITY FOR VARIOUS SCALING FACTORS

nential growth, and this brings the order of the adjoint sensitivity to match the order of the finite difference sensitivity. Further increase in the scaling factor halts the exponential growth of the energy norm of the adjoint fields and it reaches an approximate steady state level. The adjoint sensitivity in this regime agrees with the finite difference sensitivity within an error of less than $20 \%$.

Table 2 and Figure 10 show the relative error in adjoint sensitivity with respect to the finite difference sensitivity for a Gaussian shaped source term perturbation upstream of the leading edge of the vane to the conservative compressible Navier-Stokes equations. The length scale of the perturbation is $10 \mathrm{~mm}$ and it's relative magnitude to the convective term of the Navier-Stokes equation is around $1 \%$. From Table 2 it can be seen that there seems to be an optimal value of $\lambda=0.005$ and raising it beyond this point leads to a slowly increasing error in the sensitivities. 


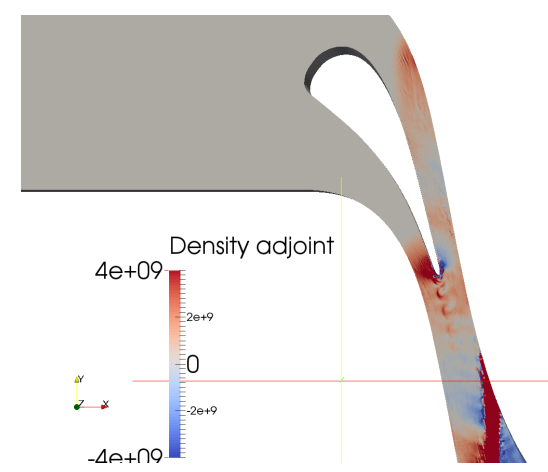

FIGURE 11. DENSITY ADJOINT SOLUTION AT $t=0$

This is due to the fact that too much viscosity is being added to the adjoint equations, making the adjoint sensitivities inaccurate. There is a sizable range of scaling factors where the adjoint flow field is sufficiently damped and the sensitivities are reasonably correct.

Figure 11 shows a visualization of the density adjoint field at $t=0$ (terminal time for the adjoint simulation). The magnitude of the adjoint field is large in the trailing edge regions, in the beginning of the wake and near the region where the averaging of the design objective is performed.

As the results show, selection of the scaling factor $\lambda$ greatly influences the adjoint sensitivities obtained. After using an initial estimate for $\lambda$ obtained from process described in the previous section multiple values of $\lambda$ need to be tried to get a set of stabilized adjoint flow simulations. Using this set Richardson extrpolation, a technique used in many numerical algorithms [23, 24], can be used to improve the accuracy of adjoint sensitivities and obtain an error estimate.

Let $g(\lambda)$ be the computed sensitivity, $g^{*}$ be the true sensitvity, representing $g$ using a polynomial expression

$$
g(\lambda)=g^{*}+a \lambda+b \lambda^{2}+\ldots+O\left(\lambda^{n}\right)
$$

Let, $g_{i+1}(\lambda)=\frac{h^{i} g_{i}(\lambda / h)-g_{i}(\lambda)}{h^{i}-1}$ with $g_{0}=g$. Then,

$$
g_{i+1}(\lambda)=g^{*}+O\left(\lambda^{i+1}\right)
$$

So, by utilizing multiple adjoint sensitivities from simulations with different scaling factors a more accurate gradient can be estimated. Though running multiple adjoint simulations is expensive, it is still much cheaper than all of the other sensitivity analysis methods for chaotic systems. This technique has the potential to make the viscous-stabilized adjoint method more useful for generic chaotic or turbulent fluid flow problems.

\section{CONCLUSION}

The unsteady adjoint method is useful for computing senstivities of objectives to a large number of parameters. The unsteady adjoint solution diverges to infinity due to chaotic dynamics of turbulence in fluid flows. An energy analysis provides valuable information about the mechanism of growth of the adjoint field. Local artificial viscosity can limit the divergence adjoint field and maintain accuracy of adjoint senstivities. The viscous stabilized unsteady adjoint method provides a promising method for computing adjoint sensitivities of long time-averaged objectives with respect to arbitrary perturbations for a turbulent fluid flow. The effectiveness of the method has been demonstrated on 2-D chaotic flow problems and will be extended to 3-D flow problems. The estimation of the optimal scaling factor for a particular fluid problem requires more work and will be investigated in the future with the help of Richardson extrapolation.

\section{ACKNOWLEDGMENT}

This research used resources of the Oak Ridge Leadership Computing Facility at Oak Ridge National Laboratory, which is supported by the Office of Science of the Department of Energy under Contract DE-AC05-00OR22725.

\section{REFERENCES}

[1] Gourdain, N., Gicquel, L. Y., and Collado, E., 2012. "Comparison of rans and les for prediction of wall heat transfer in a highly loaded turbine guide vane". Journal of Propulsion and Power, 28(2), pp. 423-433.

[2] Jameson, A., 1995. "Optimum aerodynamic design using cfd and control theory". AIAA paper, 1729, pp. 124-131.

[3] Lyu, Z., and Martins, J. R. R. A., 2014. "Aerodynamic shape optimization studies of a blended-wing-body aircraft". Journal of Aircraft, 51.

[4] Economon, T. D., Palacios, F., and Alonso, J. J., 2013. “A viscous continuous adjoint approach for the design of rotating engineering applications". AIAA Paper, 2580, pp. 2427.

[5] Wang, Q., and Gao, J.-H., 2013. "The drag-adjoint field of a circular cylinder wake at reynolds numbers 20, 100 and 500”. Journal of Fluid Mechanics, 730, pp. 145-161.

[6] Blonigan, P., Chen, R., Wang, Q., and Larsson, J., 2012. "Towards adjoint sensitivity analysis of statistics in turbulent flow simulation". In Proceedings of the Stanford Center of Turbulence Research Summer Program 2014, p. 229.

[7] Aceves, A., Adachihara, H., Jones, C., Lerman, J. C., McLaughlin, D. W., Moloney, J. V., and Newell, A. C., 1986. "Chaos and coherent structures in partial differential equations". Physica D: Nonlinear Phenomena, 18(1), pp. 85-112. 
[8] Wilcox, D. C., et al., 1998. Turbulence modeling for CFD, Vol. 2. DCW industries La Canada, CA.

[9] Arts, T., and de Rouvroit, M. L., 1992. "Aero-thermal performance of a two-dimensional highly loaded transonic turbine nozzle guide vane: A test case for inviscid and viscous flow computations". J. Turbomach., 114(1), pp. 147-154.

[10] Lea, D. J., Allen, M. R., and Haine, T. W., 2000. "Sensitivity analysis of the climate of a chaotic system". Tellus A, 52(5).

[11] Thuburn, J., 2005. "Climate sensitivities via a fokkerplanck adjoint approach". Quarterly Journal of the Royal Meteorological Society, 131(605), pp. 73-92.

[12] Ruelle, D., 2008. "Differentiation of srb states for hyperbolic flows". Ergodic Theory and Dynamical Systems, 28(02), pp. 613-631.

[13] Ruelle, D., 2009. "A review of linear response theory for general differentiable dynamical systems". Nonlinearity, 22(4), p. 855.

[14] Blonigan, P., Gomez, S., and Wang, Q., 2014. "Least squares shadowing for sensitivity analysis of turbulent fluid flows". arXiv preprint arXiv:1401.4163.

[15] Garnier, E., Adams, N., and Sagaut, P., 2009. Large eddy simulation for compressible flows. Springer Science \& Business Media.

[16] Moeng, C.-H., and Wyngaard, J. C., 1989. "Evaluation of turbulent transport and dissipation closures in second-order modeling". Journal of the Atmospheric Sciences, 46(14), pp. 2311-2330.

[17] Macdonald, C. B., 2003. "Constructing high-order rungekutta methods with embedded strong-stability-preserving pairs". PhD thesis, Simon Fraser University.

[18] Roe, P. L., 1981. "Approximate riemann solvers, parameter vectors, and difference schemes". Journal of computational physics, 43(2), pp. 357-372.

[19] Choi, H., and Moin, P., 2012. "Grid-point requirements for large eddy simulation: Chapmans estimates revisited". Physics of Fluids (1994-present), 24(1), p. 011702.

[20] Bastien, F., Lamblin, P., Pascanu, R., Bergstra, J., Goodfellow, I. J., Bergeron, A., Bouchard, N., and Bengio, Y., 2012. Theano: new features and speed improvements. Deep Learning and Unsupervised Feature Learning NIPS 2012 Workshop.

[21] Bergstra, J., Breuleux, O., Bastien, F., Lamblin, P., Pascanu, R., Desjardins, G., Turian, J., Warde-Farley, D., and Bengio, Y., 2010. "Theano: a CPU and GPU math expression compiler". In Proceedings of the Python for Scientific Computing Conference (SciPy). Oral Presentation.

[22] Abarbanel, S., and Gottlieb, D., 1981. "Optimal time splitting for two-and three-dimensional navier-stokes equations with mixed derivatives". Journal of Computational Physics, 41(1), pp. 1-33.

[23] Ford, W. F., and Sidi, A., 1987. "An algorithm for a gen- eralization of the richardson extrapolation process". SIAM journal on Numerical Analysis, 24(5), pp. 1212-1232.

[24] Celik, I., and Zhang, W.-M., 1995. "Calculation of numerical uncertainty using richardson extrapolation: application to some simple turbulent flow calculations". Journal of Fluids Engineering, 117(3), pp. 439-445. 